\title{
3D Collagen Orientation Study of the Human Cornea Using X-ray Diffraction and Femtosecond Laser Technology
}

\author{
Mohammad Abahussin, ${ }^{1,2,3}$ Sally Hayes, ${ }^{1,2}$ Nathaniel E. Knox Cartwright, ${ }^{4}$ \\ Christina S. Kamma-Lorger, ${ }^{1}$ Yasir Khan, ${ }^{4}$ John Marshall, ${ }^{4}$ and Keith M. Meek ${ }^{1}$
}

Purpose. To study the distribution and predominant orientations of fibrillar collagen at different depths throughout the entire thickness of the human cornea. This information will form the basis of a full three-dimensional reconstruction of the preferred orientations of corneal lamellae.

Methods. Femtosecond laser technology was used to delaminate the central zones of five human corneas into three separate layers (anterior, mid, and posterior stroma), each with predetermined thicknesses. Wide-angle x-ray diffraction was used to study the gross collagen fibril orientation and distribution within each layer.

Results. The middle and posterior parts of the human cornea demonstrated a preferential orthogonal arrangement of collagen fibrils, directed along the superior-inferior and nasaltemporal meridians, with an increase in the number of lamellae toward the periphery. However, the anterior cornea (33\% of total corneal thickness) showed no systematic preferred lamellar orientation.

Conclusions. In the posterior two thirds of the human cornea, collagen lies predominantly in the vertical and horizontal meridians (directed toward the four major rectus muscles), whereas collagen in the anterior third of the cornea is more isotropic. The predominantly orthogonal arrangement of collagen in the mid and posterior stroma may help to distribute strain in the cornea by allowing it to withstand the pull of the extraocular muscles, whereas the more isotropic arrangement in the anterior cornea may play an important role in the biomechanics of the cornea by resisting intraocular pressure while at the same time maintaining corneal curvature. (Invest Ophthalmol Vis Sci. 2009;50:5159-5164) DOI:10.1167/iovs.093669

From the ${ }^{1}$ School of Optometry and Vision Sciences, Cardiff University, Cardiff, United Kingdom; and 'The Rayne Institute, St. Thomas' Hospital, London, United Kingdom.

${ }^{2}$ Contributed equally to the work and therefore should be considered equivalent authors.

${ }^{3}$ Present affiliation: Optometry Department, College of Applied Medical Science, King Saud University, Riyadh, Saudi Arabia.

Supported by the U.K. Medical Research Council Grant G0600755 (KMM), the Royal College of Surgeons (YK, NC), and Abbott Medical Optics. Mohammad Abahussin was the recipient of a scholarship from King Saud University, Riyadh, Saudi Arabia. Keith Meek is a Royal Society-Wolfson Research Merit Award Holder.

Submitted for publication March 5, 2009; revised May 19, 2009; accepted August 4, 2009.

Disclosure: M. Abahussin, None; S. Hayes, None; N.E. Knox Cartwright, None; C.S. Kamma-Lorger, None; Y. Khan, None; J. Marshall, None; K.M. Meek, None

The publication costs of this article were defrayed in part by page charge payment. This article must therefore be marked "advertisement" in accordance with 18 U.S.C. $\$ 1734$ solely to indicate this fact.

Corresponding author: Keith M. Meek, School of Optometry and Vision Sciences, Cardiff University, Maindy Road, Cardiff CF24 4LU, UK; meekkm@cf.ac.uk.
$\mathrm{C}$ orneal transparency depends on the unique architecture of the stroma, which accounts for more than $90 \%$ of corneal thickness. In humans, the central stroma is made of approximately 200 to 250 stacked lamellae that lie essentially parallel to the surface of the cornea. ${ }^{1}$ Adjacent lamellae do not generally lie in the same direction, and the change in orientation between lamellae may be any angle between $0^{\circ}$ and $180^{\circ}{ }^{2-4}$ Within a single lamella, collagen fibrils of a uniform diameter lie parallel to one another. They are embedded in a hydrated matrix rich in proteoglycans and salts. ${ }^{5,6}$ The specific arrangement of collagen in the corneal stroma produces destructive interference of light scattered in all directions other than the forward direction, leading to tissue transparency. It is also responsible for maintaining the strength and shape of the cornea. $^{7,8}$

Many studies have been undertaken to determine the orientation of collagen fibrils within the human cornea. ${ }^{8-13}$ These studies indicate that, in the human cornea, there is a preferred fibril orientation in the inferior-superior and nasal-temporal directions. This preferred orientation occurs at the center of the cornea and is maintained to within $2 \mathrm{~mm}$ of the limbus, where a gradual change to a tangential disposition occurs. ${ }^{8}$ However, the collagen fibril orientation and distribution at different depths within the human cornea has yet to be investigated. In the present study, femtosecond laser technology was used to delaminate human corneas into samples at different depths within the stroma and wide-angle $x$-ray diffraction was used to study the collagen fibril orientation and distribution within each layer. This is the first step toward a full three-dimensional reconstruction of corneal lamellae preferred orientations. The results of this study should help elucidate the biomechanical origins of some problems induced by excimer laser refractive surgery.

\section{Materials ANd Methods}

\section{Samples}

All tissue used in this study was obtained in accordance with the tenets of the Declaration of Helsinki. Five human corneas with intact scleral rim were sampled. Three of them (B1-3) were obtained from the Bristol Eye Bank (Bristol, UK) and had previously been stored in culture medium (Eagle's MEM buffered with HEPES and containing $26 \mathrm{mM}$ sodium bicarbonate, $2 \%$ fetal bovine serum, $2 \mathrm{mM}$ L-glutamine, penicillin, streptomycin, and amphotericin $\mathrm{B}$ ) at $37^{\circ} \mathrm{C}$. The remaining two corneas (P1 and P2), which were obtained from a pair of donor globes provided by the National Disease Research Interchange (Philadelphia, PA), were transported to Cardiff University on dry ice. Before experimental use, the globes were allowed to thaw at room temperature and the corneas with a scleral rim were removed. Because of variations in postmortem times and storage, some corneas were swollen more than others. An ultrasonic pachymeter showed the measured corneal thickness of the five corneas to range from 570 to $1040 \mu \mathrm{m}$ (Pachette2; DGH Technology, Exton, PA). However, previous studies have shown that even severe swelling does not change the directions of corneal 
TABLE 1. Stromal Depth Location of the Delaminated Anterior, Middle, and Posterior Layers of Five Studied Corneas

\begin{tabular}{|c|c|c|c|c|c|}
\hline \multirow[b]{2}{*}{ Layer } & \multicolumn{5}{|c|}{ Cornea } \\
\hline & B1 & $\mathbf{B 2}$ & B3 & P1 & $\mathbf{P 2}$ \\
\hline Anterior & $0-120(12)$ & $0-140(14)$ & $0-160(28)$ & $0-250(36)$ & $0-200(33)$ \\
\hline Middle & $120-240(12)$ & $140-280(14)$ & $160-320(28)$ & $250-500(36)$ & $200-400(33)$ \\
\hline Posterior & $240-1040(76)$ & $280-990(72)$ & $320-570(44)$ & $500-700(28)$ & $400-600(33)$ \\
\hline Total corneal thickness & 1040 & 990 & 570 & 700 & 600 \\
\hline
\end{tabular}

Data are the distance from the surface of the cornea $(\mu \mathrm{m})$. Numbers in parentheses represent the percentage of the total corneal thickness included within each layer. Note that corneas B3, P1, and P2 were only slightly swollen compared with B1 and B2, which were swollen to almost twice physiological thickness.

lamellae within the plane of the cornea (Meek KM, et al. IOVS. 2005; 46:ARVO E-Abstract 2183). On receipt, all corneas were placed in $4 \%$ paraformaldehyde and stored at $4^{\circ} \mathrm{C}$ until required experimentally.

Each cornea was placed in a sterile Barron chamber (Katena Products Inc., Denville, NJ), and a physiologic saline solution was pumped into the posterior compartment at intraocular pressure to ensure the maintenance of a normal corneal shape and curvature during the delamination process. The chamber and cornea were then positioned under the exit point of a $60 \mathrm{kHz}$ femtosecond laser (FS 60; IntraLase Corp., Irvine, CA), and the applanation cone of the laser was brought into contact with the corneal surface, causing it to flatten. The laser parameters were set to a flap diameter of $8.5 \mathrm{~mm}$ for the anterior stromal layer and $7.5 \mathrm{~mm}$ for the mid-stromal layer, a planned flap thickness of between 120 and $250 \mu \mathrm{m}$, a side cut angle of $70^{\circ}$, a beam separation of $4 \times 4 \mu \mathrm{m}$ and a cut energy of $4 \mu \mathrm{J}$.

As shown in Table 1, the first delamination, with a diameter of 8.5 $\mathrm{mm}$, was performed between 120 and $250 \mu \mathrm{m}$ from the corneal surface, thus isolating a sample disc of the anterior stroma. Subsequently, a further delamination, of diameter $7.5 \mathrm{~mm}$, was induced at a depth of 240 to $500 \mu \mathrm{m}$ from the surface, allowing a second disc to be isolated from the mid stroma. Finally, the residual tissue, measuring between 200 and $800 \mu \mathrm{m}$ in thickness was isolated with a 12-mm trephine, providing a sample of the posterior stroma complete with a full-thickness scleral rim. Before being placed in fresh $4 \%$ paraformaldehyde, each piece of corneal tissue was marked at the 12 o'clock position by means of a small incision.

\section{X-ray Diffraction}

To minimize tissue dehydration during x-ray exposure, each piece of corneal tissue was wrapped in thin plastic film (Clingfilm; Superdrug
Stores Plc., Croydon, UK) and placed in an airtight chamber (Perspex; Databank UK, London, UK) with polyester film (Mylar; DuPont-Teijin Films, Middlesbrough, UK) windows. This was then sealed with grease. All x-ray diffraction experiments were performed on station 14.1 at the UK Synchrotron Radiation Source (Daresbury, UK), using a wide-angle beam with a wavelength of $0.1488 \mathrm{~nm}$ and a $0.2-\mathrm{mm}^{2}$ cross section of the specimen. The $\mathrm{x}$-ray exposure time differed between specimens $(25,35$, or 45 seconds) due to variations in the thickness of the corneal tissue and the intensity of the x-ray beam at the time of data collection. X-ray scatter patterns were collected at $0.5-\mathrm{mm}$ intervals over each corneal layer and recorded on a charge-coupled device (CCD) detector (Quantum 4R; ADSC, Poway, CA) located $150 \mathrm{~mm}$ behind the specimen. A lead beam stop was positioned between the sample and the detector to stop any undeviated $\mathrm{x}$-rays

Each $x$-ray scatter intensity profile was normalized against variations in $\mathrm{x}$-ray exposure time and beam intensity. Figure 1 shows the major steps of $\mathrm{x}$-ray diffraction analysis. A high-angle diffraction pattern from the central human cornea (Fig. 1A) shows the beam-stop position in the center, surrounded by a ring of x-ray scatter corresponding to equatorial scattering from the collagen molecules. Four lobes of increased $\mathrm{x}$-ray scatter intensity can also be seen that correspond to a preponderance of collagen molecules lying in the superior-inferior and nasal-temporal directions. Although the collagen molecules are inclined slightly with respect to the fibril axis, the tilt is less than $15^{\circ},{ }^{14}$ and therefore the molecular alignment can be used as a good indication of the fibril alignment.

In the first stage of the analysis, a radial background scatter (arising from noncollagenous components in the cornea) was subtracted from the reflection by taking 256 segments and fitting a power law function radially outward from the center of the x-ray scatter pattern. ${ }^{4,15}$ After

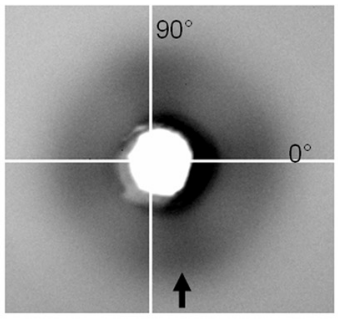

A

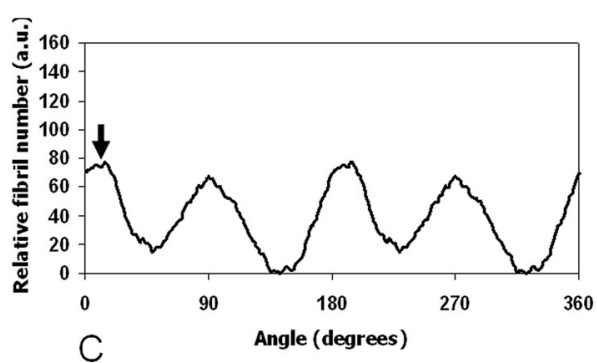

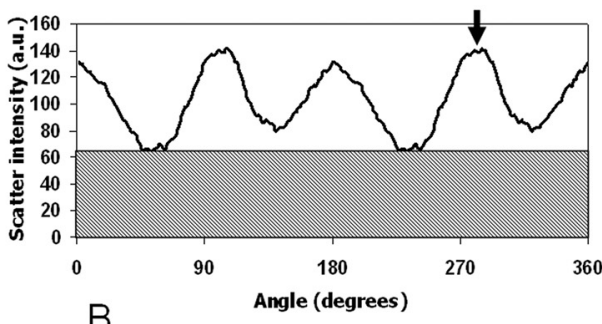

B

FIGURE 1. Main steps of $\mathrm{x}$-ray diffraction analysis. (A) A high-angle $\mathrm{x}$ ray scatter pattern recorded from the central human cornea. (B) Distribution of scatter intensity around the collagen intermolecular reflection. The total scatter can be divided into scatter from preferentially aligned collagen (clear area) and scatter from isotropically oriented collagen (shaded area). (C) Distribution of scatter intensity after the subtraction of isotropic scatter and a $90^{\circ}$ phase shift. Introducing the phase shift effectively converts (B) into a plot of relative fibril number versus angle. (D) The plot in (C) with polar coordinates. 
this, the scatter was summed for each radial position, and the summed $\mathrm{x}$-ray scatter intensity plotted as a function of angle (Fig. 1B); the total area under the scatter intensity graph is proportional to the total amount of collagen at that point in the tissue and may be subdivided into $x$-ray scatter arising from isotropically arranged collagen and scatter from preferentially aligned collagen.

Finally, the isotropic scatter was subtracted from the total scatter intensity profile, ${ }^{12,16}$ and the data shifted by $90^{\circ}$ to account for the fact that the $\mathrm{x}$-ray reflections are at right angles to the fibril axes (Fig. 1C). The remaining scatter intensity profile of preferentially aligned collagen was then plotted as polar coordinates (Fig. 1D). ${ }^{17}$ Displaying Figure 1C as a polar plot (Fig. 1D) enables the preferred fibril directions that gave rise to the $\mathrm{x}$-ray reflection in Figure $1 \mathrm{~A}$ to be clearly visualized. The radial size of the plot in a given direction is proportional to the number of fibrils disposed in that direction.

One point in the $\mathrm{x}$-ray pattern is indicated in Figure 1A (arrow) and the arrows in Figure 1B-D allow this point to be followed through each stage of the analysis.

Using this method, each $\mathrm{x}$-ray pattern was analyzed to produce a polar plot representing the amount of collagen preferentially aligned in a given direction or directions, summed throughout the full thickness of the tissue. By recording x-ray patterns across the whole tissue, an assembly of polar plots was then created to visualize the spatial distribution of preferentially aligned collagen fibrils.

To show the relative mass distribution of total and preferentially aligned collagen throughout the tissue, we devised contour maps of $\mathrm{x}$-ray scatter intensity by individually integrating the area under the total X-ray scatter intensity graph (Fig. 1B) and the area under the preferentially aligned collagen x-ray scatter graph (Fig. 1C), to produce single intensity values for each component at every sampling location. These scatter intensity values were then assimilated onto a grid relating to sampling position.

\section{Results}

The orientation of collagen fibrils in the anterior one third of cornea P2 is shown in Figure 2A. No consistent preferred orientation was detected in this anterior region $(\sim 33 \%$ of total corneal thickness). The other four corneas showed similar results in their anterior layers, and, in the case of $\mathrm{B} 1$ and $\mathrm{B} 2$, a lack of consistent collagen orientation was also seen in the mid-stromal layer of each cornea. However, the results obtained from the mid and posterior stromal layers of corneas B1 and B2 should be treated with caution due to the swollen nature of the tissue. Although, as previously stated, stromal swelling does not affect the predominant orientation of collagen (within the plane of the tissue) (Meek KM, et al. IOVS 2005;46:ARVO E-Abstract 2183), it may alter the relative proportion of fibrillar collagen present in the mid- and posterior stromal layers and explain the lack of detection of any preferred orientation in the mid-stroma of these corneas.

Figure 2B shows the collagen fibril orientation in the middle layer of cornea P2 (200-400 $\mu \mathrm{m}$ from the corneal surface). Collagen fibrils were preferentially aligned in orthogonal directions throughout the $7.5-\mathrm{mm}$ corneal disc and the amount of preferentially aligned collagen appeared to increase toward the periphery.

Figure 2C shows the predominant orientation of collagen in the posterior $200 \mu \mathrm{m}$ of cornea P2 and in its full thickness scleral rim. The relative size of the polar plots (as shown by the color key) is greater in this layer than in the anterior and middle layers, indicating proportionally more aligned collagen in this region of the tissue. The collagen arrangement seen in the central 8 to $9 \mathrm{~mm}$ of the posterior cornea was similar to that found in previous $x$-ray scattering studies of full-thickness corneas. $^{8,10,12,18,19}$ Consistent with previous studies of whole corneas, the orthogonal orientation of collagen fibrils in the central cornea was gradually replaced with a tangential arrangement near the limbus and the amount of aligned collagen increased with distance from the center. ${ }^{4,8,15,19}$

Figure 3 shows the distribution of collagen fibrils in the anterior, middle, and posterior layers of cornea P2. Figures $3 \mathrm{~A}$, $3 \mathrm{C}$, and $3 \mathrm{E}$ all indicate that the total scatter from fibrillar collagen remained fairly constant in the central $3 \mathrm{~mm}$ of the cornea (yellow/orange contours) but increased toward the periphery (red/black contours). This finding was expected, as all three layers were of a similar tissue thickness $(200 \mu \mathrm{m})$. The increase in total collagen content observed in the posterior stromal layer, beyond the region from which the anterior and mid stromal discs were removed (Fig. 3E), was the result of $\mathrm{x}$-ray scattering data being gathered at full tissue thickness.

Scatter from preferentially aligned collagen in the anterior, middle, and posterior layers (cornea P2) is shown in Figures $3 \mathrm{~B}, 3 \mathrm{D}$, and $3 \mathrm{~F}$. The anterior layer has less aligned collagen than the middle layer, which in turn has less than the posterior layer, as indicated by the colors of the contours. In the posterior layer (Fig. 3F), the diamond shape seen in normal fullthickness corneas ${ }^{8,19}$ can also be seen here encroaching on the central region, particularly in the top right quadrant at the 2 o'clock position. This feature has been postulated to arise from a system of anchoring lamellae that traverse the peripheral cornea, and it would seem from this result that these lamellae are located in the posterior third of the cornea, although further research will be needed to confirm this.

Table 2 shows the proportion of collagen lamellae (within the plane of the stroma) that are preferentially oriented (over and above a population of collagen fibrils oriented equally in all directions), in the central $7 \mathrm{~mm}$ of the anterior, middle, and posterior regions of three corneas of comparable thickness (B3, P1, and P2). The proportion of aligned collagen at each tissue depth was calculated by dividing the intensity of $\mathrm{x}$-ray scatter from preferentially aligned collagen at each sample point by the total $x$-ray scatter intensity at that point (expressed as a percentage) and then averaging the calculated values over the central $7-\mathrm{mm}$ region. It is clear that the proportion of preferentially oriented collagen increases throughout the thickness of the tissue.

\section{Discussion}

For the first time, the predominant orientation and relative mass distribution of collagen at different depths throughout the human cornea has been revealed. The results presented in this article indicate that the collagen fibrils are proportionally more aligned in the posterior two thirds of the cornea whereas in the anterior third, the orientation of collagen is more isotropic.

Most previous x-ray scattering studies of corneal collagen orientation have been performed on full-thickness corneas. ${ }^{8,19-21}$ Electron microscopy, on the other hand, has provided information on the arrangement of the collagen fibrils at different corneal depths, but it has not been so successful in determining larger scale orientation ${ }^{11}$; furthermore, considerable variation appears to exists between studies. This variation has been attributed to methodological factors, mainly tissue preparation. $^{22-24}$ With the evolution of femtosecond laser technology, a human cornea can now be delaminated into separate uniform layers of predetermined thicknesses with minimal tissue alteration. ${ }^{25-27}$ Using this method to accurately separate the cornea into anterior, middle, and posterior layers, and $\mathrm{x}$-ray scattering techniques to determine the precise orientation of collagen and its relative mass distribution at specific points within each layer, we have been able to determine the gross organization of collagen fibrils throughout the thickness of the human cornea. 


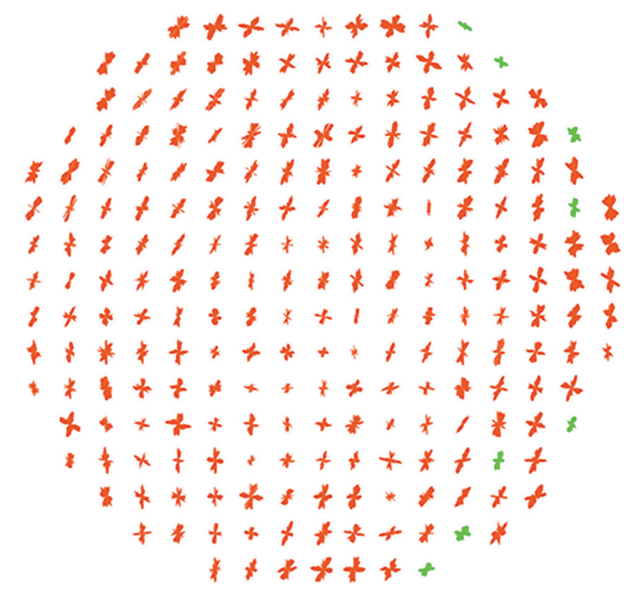

(A) ANTERIOR

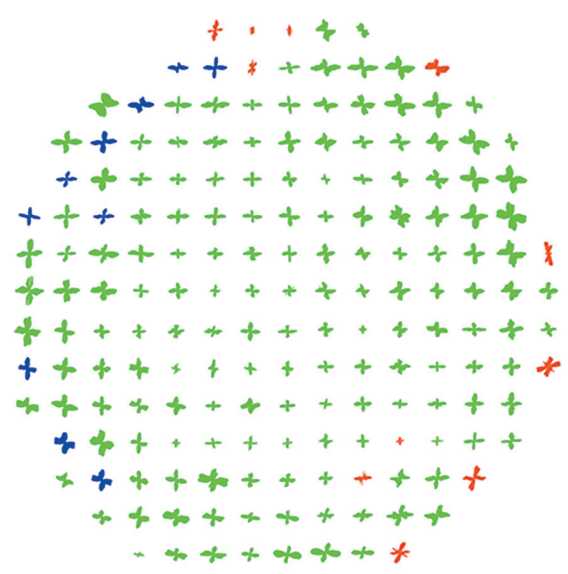

(B) MIDDLE

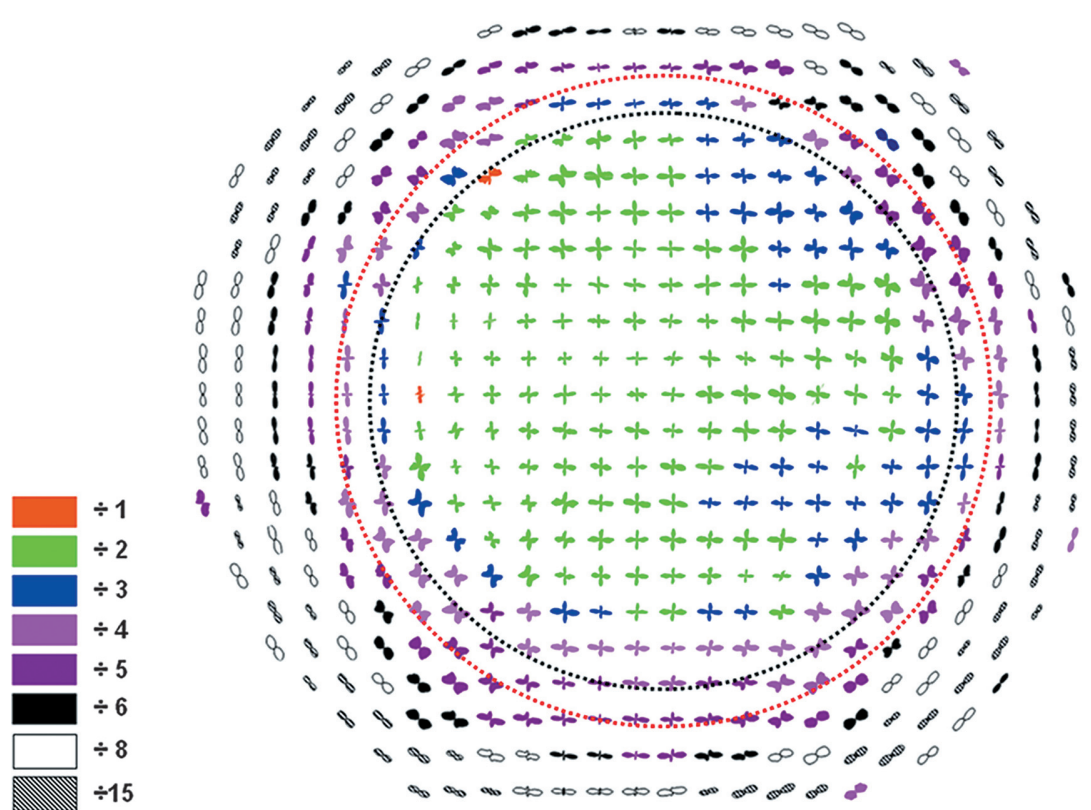

(C) POSTERIOR
FigURE 2. Polar plot maps showing collagen fibril orientation at $0.5-\mathrm{mm}$ intervals across the anterior third (A) and middle $200 \mu \mathrm{m}$ region (B) of a human cornea (P2). The posterior $200 \mu \mathrm{m}$ of the cornea (with a fullthickness scleral rim) (C), is shown after removal of the anterior (red dotted line) and middle (black dotted line) layers from the central 8- to 9-mm region. Polar plots have been scaled down to fit onto the grid as indicated in the color key.
$6 \mathrm{~mm}$

Omm the limbus. ${ }^{4,9,15}$ Transmission and scanning electron microscopy studies showed that collagen lamellae in the anterior one third of the stroma are narrower and more irregularly interwoven than are those in the deeper two thirds of the cornea. ${ }^{1,11,28-29}$ Many of these anterior lamellae insert into Bowman's layer, as has been elegantly demonstrated using second harmonic imaging confocal microscopy. ${ }^{30}$

The results of the present study are in agreement with previous studies and show an isotropic orientation of collagen fibrils in the first $200 \mu \mathrm{m}$ of the cornea. Previous depth studies have been limited to the central cornea, ${ }^{10,30}$ whereas in this study, both the central and paracentral regions of the cornea were examined. However, due to the maximum 9-mm cut diameter of the femtosecond laser, it was not possible to determine the collagen fibril orientation as a function of tissue depth around the limbus. As shown in Table 1, cuts were made at different tissue depths in each cornea to pinpoint the precise location at which the observed orthogonal preferred orienta- 
Figure 3. Contour maps showing the intensity of $x$-ray scatter (arbitrary units) from total fibrillar collagen $(\mathbf{A}, \mathbf{C}, \mathbf{E})$ and preferentially aligned collagen $(\mathbf{B}, \mathbf{D}, \mathbf{F})$ in the anterior $(\mathbf{A}, \mathbf{B})$ and middle $(\mathbf{C}, \mathbf{D})$ stroma of cornea P2 and in the remaining posterior cornea with scleral $\operatorname{rim}(\mathbf{E}, \mathbf{F})$. Dotted white line: The central 8- to 9-mm region of the cornea from which the anterior and middle layers were removed.
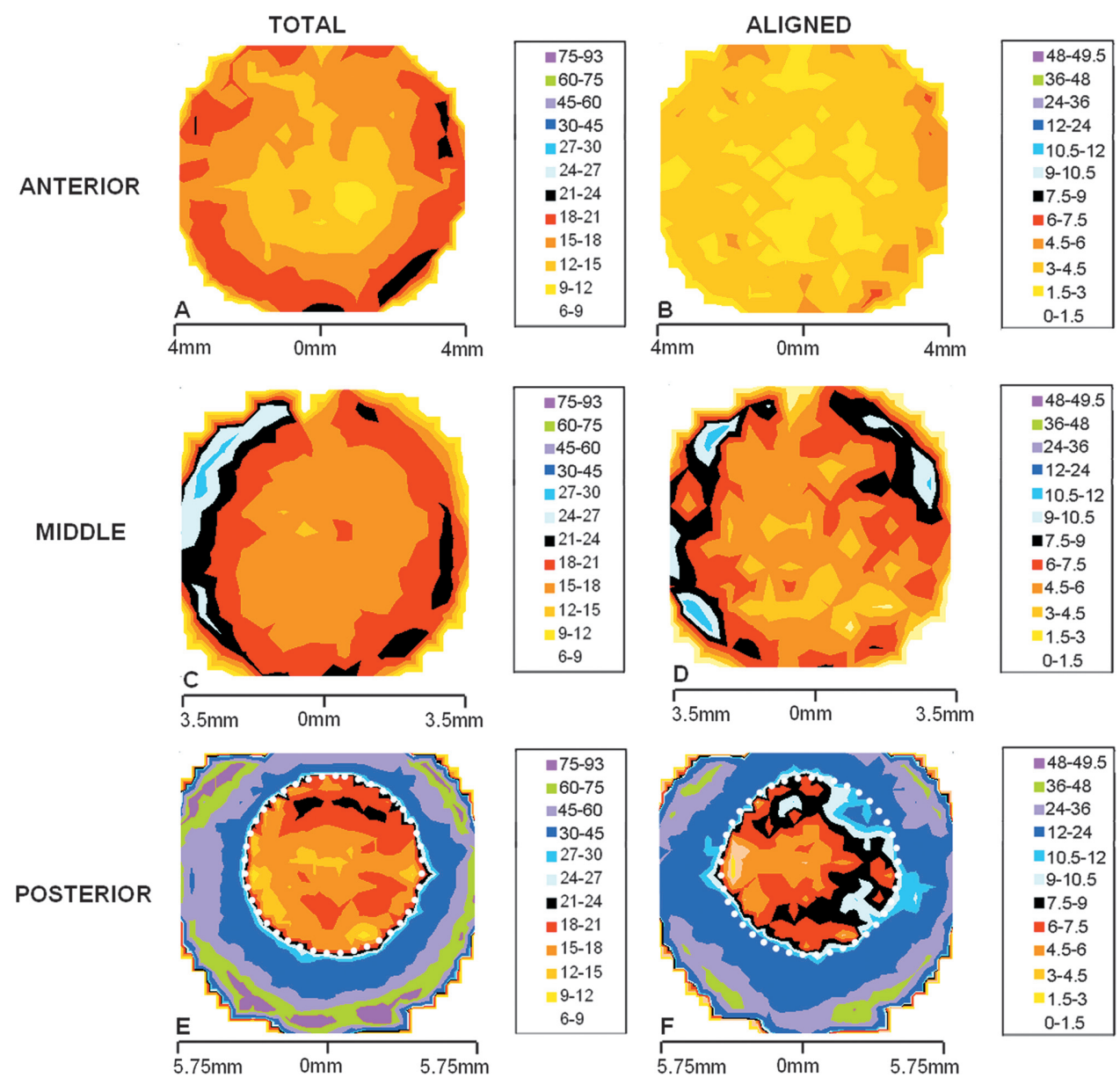

tion of collagen predominates. Analysis of the x-ray scattering data showed that this arrangement of collagen began beyond the anterior third of the cornea.

The irregular fibril orientation in the anterior stroma was most pronounced in the central $4 \mathrm{~mm}$ area. Beyond that, some lamellae were found to be aligned in orthogonal directions, as demonstrated by the presence of cross-shaped plots toward the periphery in Figure 2. More orthogonally oriented lamellae also occurred away from the center in the middle stroma (Fig. 2B) as evidenced by the increase in the size of the plots. Since the femtosecond laser's mode of operation allows cuts to be achieved at predetermined depths with an accuracy of \pm 14 $\mu \mathrm{m}^{25}$ and the damage to surrounding tissues is minimal, ${ }^{26}$ no significant changes to collagen lamellae are likely to have been induced a result of the isolation process. The observed increase in collagen scatter toward the peripheral cornea is therefore unlikely to have been caused by differences in section thickness and was probably caused by changes in collagen density or hydration, both of which affect x-ray scatter intensity.

TABLE 2. The Percentage of the Total Collagen Fibrils That Were Preferentially Aligned in the Anterior, Middle, and Posterior Layers of Three Human Corneas

\begin{tabular}{lcccc}
\hline & \multicolumn{3}{c}{ Cornea } & \\
\cline { 2 - 4 } Layer & B3 & P1 & P2 & Average \\
\hline Anterior & 24 & 22 & 21 & 22 \\
Middle & 30 & 32 & 31 & 31 \\
Posterior & 41 & 45 & 41 & 42 \\
\hline
\end{tabular}

In addition to polar plots, contour maps showing the relative distribution of aligned collagen mass quantitatively support the finding that the anterior third of the cornea is less aligned collagen than the posterior two thirds. This finding is further illustrated when the amount of preferentially aligned collagen in each layer is shown as a percentage of the total collagen (Table 2 ). In the posterior stroma, $42 \%$ of the lamellae are preferentially aligned in the vertical or horizontal direction ( $\sim 21 \%$ in each direction), leaving $58 \%$ isotropically arranged. This means that, in the posterior stroma, the vertical and horizontal directions are reinforced significantly compared with other directions. In the anterior stroma, this value drops to $11 \%$ in each direction. Biomechanical strip testing has revealed that full thickness strips cut from the vertical and horizontal directions of human corneas are between $13 \%$ and $25 \%$ stronger than strips from intermediate directions. ${ }^{31}$ Our results suggest that the additional strength comes from lamellae in the posterior corneal stroma.

A further interesting observation made in this study was that the skewed diamond-shaped arrangement seen previously in contour maps of aligned collagen distribution, which is thought to be due to the presence of additional lamellae that traverse the peripheral cornea, ${ }^{8,19}$ seems to occur only in the posterior region of the cornea. The posterior third of the cornea clearly has an interesting, nonisotropic, lamellar organization that may extend into the peripheral region and the limbus and thus warrants further investigation.

Current popular refractive surgeries involve the ablation of corneal lamellae. These surgeries have been improved using a fairly empiric approach and complex assumptions, and as a result, the outcomes of refractive surgery may, and sometimes 
do, differ from those expected. Part of this results from a limited understanding of the organization of collagen fibrils in the corne $\mathrm{a}^{32}$ and part because of similar problems with wound healing. Currently, finite element models are being developed to simulate the biomechanical response of the cornea, in an attempt to more accurately predict the result of refractive surgery in terms of shape and astigmatism. It is believed that the quantitative information presented herein may be helpful for improving understanding of the biomechanical properties of the cornea and may add further details about collagen fibril organization to computational finite element models. ${ }^{33,34}$

\section{Acknowledgments}

The authors thank IntraLase for the loan and use of its femtosecond laser; Sheraz Daya, Saj Khan, and Damian Lake from the Centre for Sight (East Grinstead, UK) for their invaluable help with the femtosecond laser; Mike McDonald (Synchrotron Radiation Source, Daresbury, UK) for assistance during data collection; Craig Boote (Cardiff University, UK) for advice and expertise in $\mathrm{x}$-ray data analysis; Valerie Smith (Bristol Eye Bank, UK) and the National Disease Research Interchange (Philadelphia, PA) for provision of the human corneas.

\section{References}

1. Hogan MJ, Alvarado JA, Weddell JE. The cornea. Histology of the Human Eye. Philadelphia, W.B. Saunders Company. 1971:55-111.

2. Maurice DM. The cornea and sclera. In: Davson H. The Eye. London: Academic Press; 1969:489-600.

3. Radner W, Zehetmayer M, Aufreiter R, Mallinger R. Interlacing and cross-angle distribution of collagen lamellae in the human cornea. Cornea. 1998; 17:537-543.

4. Newton RH, Meek KM. The integration of the corneal and limbal fibrils in the human eye. Biophys J. 1998;75:2508-2512.

5. Quantock AJ, Meek KM, Chakravarti S. An X-ray diffraction investigation of corneal structure in lumican-deficient mice. Invest $O p h$ thalmol Vis Sci. 2001;42:1750-1756.

6. Meek KM, Dennis S, Khan S. Changes in the refractive index of the stroma and its extrafibrillar matrix when the cornea swells. Biophys J. 2003;85:2205-2212.

7. Farrell R, McCally R. Corneal transparency. In: Albert D, Jakobiec F. Principles and Practice of Ophthalmology. Philadelphia: Saunders; 2000:629-643.

8. Aghamohammadzadeh $\mathrm{H}$, Newton $\mathrm{RH}$, Meek KM. X-ray scattering used to map the preferred collagen orientation in the human cornea and limbus. Structure. 2004;12:249-256.

9. Kokott W. Ubermechanisch-funktionelle strikturen des auges. Albrecht von Graefes Arch Ophthalmol. 1938;138:424-485.

10. Meek KM, Blamires T, Elliott GF, Gyi TJ, Nave C. The organisation of collagen fibrils in the human corneal stroma: a synchrotron X-ray diffraction study. Curr Eye Res. 1987;6:841-846.

11. Komai Y, Ushiki T. The three-dimensional organization of collagen fibrils in the human cornea and sclera. Invest Ophthalmol Vis Sci. 1991;32:2244-2258.

12. Daxer A, Fratzl P. Collagen fibril orientation in the human corneal stroma and its implication in keratoconus. Invest Ophthalmol Vis Sci. 1997;38:121-129.

13. Misson GP. Circular polarization biomicroscopy: a method for determining human corneal stromal lamellar organization in vivo. Ophthalmic Physiol Opt. 2007;27:256-264.
14. Holmes DF, Gilpin CJ, Baldock C, Ziese U, Koster AJ, Kadler KE. Corneal collagen fibril structure in three dimensions: structural insights into fibril assembly, mechanical properties, and tissue organization. Proc Natl Acad Sci U S A. 2008;98:7307-7312.

15. Newton RH, Meek KM. Circumcorneal annulus of collagen fibrils in the human limbus. Invest Ophthalmol Vis Sci. 1998;39:11251134.

16. Boote C, Dennis S, Meek KM. Spatial mapping of collagen fibril organisation in primate cornea: an X-ray diffraction investigation. $J$ Struct Biol. 2004;146:359-367.

17. Connon CJ, Meek KM. Organization of corneal collagen fibrils during the healing of trephined wounds in rabbits. Wound Repair Regen. 2003;11:71-78.

18. Boote C, Dennis S, Huang Y, Quantock AJ, Meek KM. Lamellar orientation in human cornea in relation to mechanical properties. J Struct Biol. 2005;149:1-6.

19. Boote C, Hayes S, Abahussin M, Meek KM. Mapping collagen organization in the human cornea: left and right eyes are structurally distinct. Invest Ophthalmol Vis Sci. 2006;47:901-908.

20. Meek KM, Quantock AJ. The use of X-ray scattering techniques to determine corneal ultrastructure. Prog Retin Eye Res. 2001;20:95137.

21. Meek KM, Tuft SJ, Huang Y, et al. Changes in collagen orientation and distribution in keratoconus corneas. Invest Ophthalmol Vis Sci. 2005; 46:1948-1956.

22. Freund DE, McCally RL, Farrell RA, Cristol SM, L'Hernault NL, Edelhauser HF. Ultrastructure in anterior and posterior stroma of perfused human and rabbit corneas: relation to transparency. Invest Ophthalmol Vis Sci. 1995;36:1508-1523.

23. Daxer A, Misof K, Grabner B, Ettl A, Fratzl P. Collagen fibrils in the human corneal stroma: structure and aging. Invest Ophthalmol Vis Sci. 1998;39:644-648.

24. Meek KM, Fullwood NJ. Corneal and scleral collagens-a microscopist's perspective. Micron. 2001;32:261-272.

25. Kezirian GM, Stonecipher KG. Comparison of the IntraLase femtosecond laser and mechanical keratomes for laser in situ keratomileusis. J Cataract Refract Surg. 2004;30:971-978.

26. Holzer MP, Rabsilber TM, Auffarth GU. Femtosecond laser-assisted corneal flap cuts: morphology, accuracy and histopathology. Invest Ophthalmology Vis Sci. 2006;47:2828-2831.

27. Sarayba MA, Ignacio TS, Tran DB, Binder PS. A $60 \mathrm{kHz}$ IntraLase femtosecond laser creates a smoother LASIK stromal bed surface compared to a Zyoptix XP mechanical microkeratome in human donor eyes. J Refract Surg. 2007;23:331-337.

28. Davson H. The Eye. Vol 1b. 3rd ed. New York: Academic Press; 1984.

29. Klyce SD, Beuerman RW. Structure and function of the cornea. In: Kaufmann HE, ed. The Cornea. New York: Churchill Livingstone; 1988:3-28

30. Morishige N, Wahlert AJ, Kenney MC, et al. Second-harmonic imaging microscopy of normal human and keratoconus cornea. Invest Ophthalmol Vis Sci. 2007;48:1087-1094.

31. ElSheikh A, Brown M, Alhasso D, Rama P, Campanelli M, GarwayHeath D. Experimental assessment of corneal anisotropy.J Refract Surg. 2008;24:178-187.

32. Fullwood NJ. Collagen fibril orientation and corneal curvature. Structure. 2004;12:169-170.

33. Pinsky PM, Van der Heide D, Chernyak D. Computational modelling of mechanical anisotropy in the cornea and sclera. J Cataract Refract Surg. 2005;31:136-145.

34. Lanchares E, Calvo B, Cristobal JA, Doblare M. Finite element simulation of arcuates for astigmatism correction. J Biomech. 2008;41:797-805. 